\title{
Simulated shifts in trophic niche breadth modulate range loss of alpine butterflies under climate change
}

\author{
Patrice Descombes ${ }^{\dagger}$, Jean-Nicolas Pradervand ${ }^{\dagger}$, Joaquim Golay, Antoine Guisan and Loïc Pellissier ${ }^{\ddagger}$ \\ P. Descombes and L. Pellissier (loic.pellissier@usys.ethz.ch), Univ. of Fribourg, Unit of Ecology and Evolution, Ch. du Musée 10, CH-1700 \\ Fribourg, Switzerland, and Landscape Ecology, Inst. of Terrestrial Ecosystems, ETH Zürich, Zürich, Switzerland, and Swiss Federal Research \\ Institute WSL, CH-8903 Birmensdorf, Switzerland. - J.-N. Pradervand, J. Golay and A. Guisan, Dept of Ecology and Evolution, Univ. of \\ Lausanne, Bâtiment Biophore, CH-1015 Lausanne, Switzerland. AG also at: Inst. of Earth Surface Dynamics, Univ. of Lausanne, CH-1015 \\ Lausanne, Switzerland.
}

\begin{abstract}
Species currently track suitable abiotic and biotic conditions under ongoing climate change. Adjustments of trophic interactions may provide a mechanism for population persistence, an option that is rarely included in model projections. Here, we model the future distribution, of butterflies in the western Alps of Switzerland under climate change, simulating potential diet expansion resulting from adaptive behavior or new host opportunities. We projected the distribution of 60 butterfly and 298 plant species with species distribution models (SDMs) under three climate change scenarios. From known host plants, we allowed a potential diet expansion based on phylogenetic constraints. We assessed whether diet expansion could reduce the rate of expected regional species extinction under climate change. We found that the risk of species extinctions decreased with a concave upward decreasing shape when expanding the host plant range. A diet expansion to even a few phylogenetically closely related host plants would significantly decrease extinction rates. Yet, even when considering expansion toward all plant species available in the study area, the overall regional extinction risk would remain high. Ecological or evolutionary shifts to new host plants may attenuate extinction risk, but the severe decline of suitable abiotic conditions is still expected to drive many species to local extinction.
\end{abstract}

Species currently respond to climate change by tracking favorable environments in the landscape (Parmesan 2006, Walther et al. 2010, Schweiger et al. 2012). The assumption that species are bound to particular environmental conditions fostered the development of species distribution models (SDMs). These models fit a multidimensional niche volume from correlations between occurrence and environmental conditions and use it to project species distributions (Guisan and Thuiller 2005). By changing the input climatic maps in the models, the projections of future colonization and extinction events under climate change can be derived (Thuiller et al. 2005, Pradervand et al. 2014). However, accumulating evidence indicates that evolutionary or ecological changes may modify how species respond to environmental conditions over very few generations (Thuiller et al. 2013a). An ecological or evolutionary adaptive response may thus be a possible alternative mechanism for population rescue under climate change (Pateman et al. 2012). It is not clear whether adaptive changes can operate fast enough to prevent species from extinction. Because of this uncertainty, it may be important to include adaptation potential scenarios, such as changes in biotic interactions, into climate change impact assessments. The combination of these scenarios with SDMs has been proposed (Thuiller et al. 2013a), but rarely used to assess species sensitivity to climate change (but see
Brooker et al. 2007, Kearney et al. 2009, Van der Putten et al. 2010).

Many herbivores, such as butterfly, are shifting their distribution poleward (Devictor et al. 2012) and to higher elevation in response to climate change. Species have moved upward with an elevational shift of up to $300 \mathrm{~m}$ from 1967-2005 (Wilson et al. 2007, Merrill et al. 2008), and projections under expected climate change suggest further elevational shifts of $650 \mathrm{~m}$ by 2100 (Merrill et al. 2008). In addition, due to shrinking suitable areas, as much as $60 \%$ of the species may be lost in some mountain ranges (Thuiller et al. 2005, Engler et al. 2011). However, SDMs are criticized because they are often an oversimplified estimation of species distribution, as they may only consider abiotic factors in future forecasts (Guisan and Thuiller 2005, Wisz et al. 2013). By contrast, evidence indicates that biotic interactions, especially trophic interactions, can affect species distributions under climate change (Araújo and Luoto 2007, Pellissier et al. 2012a, b, Schweiger et al. 2012, Eskildsen et al. 2015). In addition, microevolution could mitigate the effects of climate change. Process-based models of tree phenology incorporating the divergence of phenological responses across species ranges predict less-severe shifts in species distribution in response to climate change than niche-based models (Morin and Chuine 2006, Morin and

†Shared first authorship, ${ }^{\ddagger}$ shared last authorship 
Thuiller 2009). While biotic interactions in general should be included when modeling the response of species to climate change (Nogués-Bravo and Rahbek 2011, Kissling et al. 2012, Wisz et al. 2013), potential shifts in biotic interactions should also be considered.

Under climate change, evolutionary responses may especially modulate biotic interactions (Lavergne et al. 2010). For example, climate change may induce new interactions between species that never co-occurred or evolutionary shifts in species traits modulation interactions (Reznick and Ghalambor 2001, Gilman et al. 2010, Lavergne et al. 2010). Herbivores are frequently specialized on a restricted range of host plants, as they require detoxification mechanisms to digest plant material containing secondary metabolites (Pellissier et al. 2013a, b, Rasmann et al. 2014). Restricted host plant ranges are thought to reduce the ability of herbivores to colonize new geographical regions, especially if their hosts are rare or patchily distributed in the landscape (Pöyry et al. 2009). As a consequence, the possibility of partial release from biotic constraints via host-plant shifts may allow species to better track changing climates in the landscape and increase survival under climate change. For instance, Pateman et al. (2012) documented that the evolution of a larger larval host-plant range of the butterfly Aricia agestis has facilitated rapid range expansion under climate change. Diet expansion can be the result of evolutionary responses (i.e. adaptation to new chemical compounds that broaden the diet to new plant clades) or the result of new, previously pre-adapted herbivory opportunities (i.e. sharing the same chemical compounds as the host plant). The constraint of trophic interactions on species responses to climate change is well documented (Araújo and Luoto 2007, Schweiger et al. 2010, 2012, Romo et al. 2014, Eskildsen et al. 2015). Yet, it remains unknown how changes in herbivores trophic regimes may modify species responses to climate change.

One possible approach is to simulate shifts in diet breadth and assess the degree of diet expansion required to rescue an herbivore species pool from climate change. Here, we used high-resolution maps of environmental predictors derived from weather stations and three IPCC-based climate change scenarios to model the response of a group of specialist herbivores (butterflies) to future warming in mountain region. The goals of the study were to investigate 1) the constrain of trophic interactions on the future distribution of a regional butterfly fauna relative to abiotic conditions and 2) in which manner host plant expansion could decrease extinction risks under climate change.

\section{Material and methods}

\section{Study area and data collection}

The study area is located in the western Alps of Switzerland (Supplementary material Appendix 1, Fig. A1) and encompasses a wide elevational gradient (375 to $3200 \mathrm{~m}$ a.s.l.). The species data were collected using a random stratified sampling method (Hirzel and Guisan 2002) based on slope, elevation and exposure. The vegetation sampling included 912 sites surveyed exhaustively in $2 \times 2 \mathrm{~m}$ squares across the entire gradient (Dubuis et al. 2011), whereas the butterflies were sampled in a subset of 192 of these sites from 1000 to $3200 \mathrm{~m}$, employing the same sampling strategy (Pellissier et al. 2013a, b). Butterflies were sampled in $50 \times 50 \mathrm{~m}$ plots during summer 2009 and 2010 when the conditions were optimal (i.e. low wind, minimum temperature of $18^{\circ} \mathrm{C}$, and between 10:00 and 17:00 during the hours of high butterfly activity; Pollard and Yates 1993).

\section{Environmental predictor variables}

To model plant and butterfly species distributions, we used predictors known to have a strong influence on species distributions and we considered only variables with low correlation $(<0.7)$ to avoid collinearity. We selected the climatic variables based on biological knowledge of the target groups (Boggs and Inouye 2012, Roland and Matter 2013), their use in previous studies (Engler and Guisan 2009, Pellissier et al. 2012a, b) and a preliminary analysis of variable importance in the models (Supplementary material Appendix 1, Fig. A2). To model plant distribution, we used the temperature of the growing season (averaged from July to September), winter precipitation (averaged from January to March) representing snow cover, solar radiation, slope and topographic position as proxies for soil and wetness conditions (Engler and Guisan 2009, Randin et al. 2009). Soil variables, although important for plants (Dubuis et al. 2013), were not included as no spatially-explicit map is available for the study area. To model butterfly distribution, we used temperatures of the growing season, solar radiation as energy influx for larval growth and winter precipitation as snow cover indicator (Boggs and Inouye 2012). The temperature and precipitation values were obtained by relating information from weather stations to elevation as described in Zimmermann and Kienast (1999). For the current conditions, we used the 1981-2009 average that corresponds to the reference period used by the Centre for Climate System Modelling ( $<$ www.c2sm.ethz.ch $/>$ ). We computed the total amount of solar radiation (direct + diffuse + reflected) received by each pixel for the growing season using the spatial analyst tool in ArcGIS 10 (ESRI). We calculated the slope (degree) and the topographic position (degree of convexity/ concavity) with the spatial analyst tool in ArcGIS. Variables were computed at a resolution of $25 \mathrm{~m}$ to model plant species (558 452 pixels) and $50 \mathrm{~m}$ to model butterfly species (139 613 pixels), representing a total surface of $349 \mathrm{~km}^{2}$ (Supplementary material Appendix 1, Fig. A3).

\section{Species distribution models}

We modeled the distribution of 298 plant and 60 butterfly species presenting a minimum of 10 presences and absences in the study area. To model species distributions, we used three common statistical techniques shown to provide efficient predictions of species distributions (Elith et al. 2006): generalized linear models (GLMs; McCullagh and Nelder 1989), generalized additive models (GAMs; Hastie and Tibshirani 1990), and gradient boosting machines (GBMs; Ridgeway 1999, Friedman 2001). All the models were computed using the biomod $2 \mathrm{R}$ package with parameters optimized for species distribution modeling (Thuiller et al. 
2013b). For model validation, we used the area under the ROC-plot curve (AUC; Hanley and Mcneil 1982, Fielding and Bell 1997) and the true skill statistic (TSS; Allouche et al. 2006), which evaluate the ability of the model to discriminate presences from absences. Models are considered to have reliable prediction performances with AUC values $>0.7$ and TSS values $>0.4$ (Thuiller et al. 2009). Data were split randomly into two partitions: $80 \%$ was used for model calibration, and the remaining $20 \%$ was used for model evaluation. This procedure was replicated 10 times. We then averaged all the model projections presenting an AUC value $>0.7$ to build a total consensus binary map using the sensitivity $=$ specificity binarization criteria (Liu et al. 2005). It is expected that the accuracy of the model may vary with species traits (e.g. dispersal; Eskildsen et al. $2013,2015)$. We only considered those species with at least one model with good predictive abilities (AUC $>0.7$ ), excluding several species with long dispersal abilities potentially not associated with local habitats during the monitoring (Geiger 1987). The variable importance in the models was calculated as one minus the correlation of the original model and the model with the given variable randomized (Thuiller et al. 2013b).

\section{Climate change scenarios}

We used three different climate projection scenarios (RCP3PD, A1B and A2) averaged for three climatic models (ARPEGE-ALADIN, ECHAM5-REMO, HadCM3Q0CLM). We used a unique averaged future projection time period (i.e. 2085 from the 2070-2099) developed in the Swiss Climate Change Scenario CH2011 project from the Center for Climate Systems Modeling (<www.c2sm.ethz. $\mathrm{ch} />$ ). They are based on new generations of climate models at high resolution combining global and regional models (Bosshard et al. 2011). These climatic anomalies for every weather station in Switzerland were interpolated using the same approach as the climatic data and then added to the maps of the current climate. We projected all the plant and butterfly species distributions for the three different scenarios. We assumed unlimited dispersal of species shown to be a close approximation to using dispersal kernels in mountains (Engler and Guisan 2009).

\section{Butterfly interaction with host plants}

We compiled a list of host plants for each of the butterfly species considered (see Pellissier et al. 2012a, b, 2013a, b for further details). Phylogenetic conservatism in plant defense traits implies that closely related species produce similar secondary compound classes and require similar detoxification mechanisms in herbivores (Becerra 1997). Herbivores are thus more likely to enlarge their diet to closely related host plant species. To support this assumption, we tested whether the phylogenetic distance between the host plants of each butterfly species was lower than expected by chance using the 'ses.mpd' function in the 'picante' package with a tip label randomization (Kembel et al. 2010). Phylogenetic clustering in host plant choice would support the use of simulating diet expansion based on phylogenetic relationships.

\section{Diet expansion simulations}

We considered two approaches to simulate diet expansion. First, we inferred the probability of trophic interaction between butterfly and plant species pairs using a statistical latent trait model (Pellissier et al. 2013a, b). Probabilities of interaction between butterfly and plant species are derived from the observed matrix of trophic interactions and related to plant traits and both butterfly (Pellissier et al. 2012a, b) and plant phylogenies (Ndiribe et al. 2013). The model is then extrapolated to the entire butterfly plant network to obtain a matrix of interaction probabilities for each butterfly and plant species pair (see Pellissier et al. 2013a, b for further details). The potential trophic expansion can then be simulated by changing the interaction binary threshold in the linkage probability matrix (e.g. a threshold of 0.9 only allows interacting pairs with probabilities $>0.9$ ). Second, we simulated diet expansion directly along a phylogenetic axis without the use of a statistical model. For each butterfly species, host plant range was broadened along a scale from zero to the most distant species pairs (i.e. 270 myr) with a 1 myr step resolution (e.g. a threshold of 10 myr only allows interacting host plants which are at a phylogenetic distance $<10$ myr).

To account for the obligate trophic interaction between the butterfly and its host plants, we filtered the binary maps of the butterfly projections by those of the host plants as performed by Schweiger et al. (2008, 2012, Supplementary material Appendix 1, Fig. A4). We computed the number of species gaining or losing occupied surface under climate change and the number of species at risk of extinction along varying diet expansion thresholds. Butterfly species were considered as vulnerable to extinction when their range decreased below a threshold of $5 \%$ of the total available open habitats (i.e. total open habitats $=349 \mathrm{~km}^{2} ; 5 \%$ of total open habitats $<17.5 \mathrm{~km}^{2}$, results for thresholds of 1 and $10 \%$ available in Supplementary material Appendix 1).

\section{Results}

\section{Model performance}

Most of the plant distribution models were reliable with mean AUC values of $0.860 \pm 0.050$ and mean TSS values of $0.685 \pm 0.099$. All 298 plant species had at least one model with AUC > 0.7, except Cerastium arvense. Butterfly SDMs had a mean AUC value of $0.799 \pm 0.081$ and mean TSS values of $0.615 \pm 0.154$. Several species such as Papilio machaon showed weak predictive abilities with the three modelling techniques used. We considered a final subset of 60 butterflies with at least one good model among the three statistical techniques (Supplementary material Appendix 1, Table A1). The examination of climatic variables showed that the average winter precipitation and average summer temperature were the most important variables in the models for plant, and butterflies (Supplementary material Appendix 1, Fig. A5).

\section{Phylogenetic structure in host plant use}

We found that butterfly species with more than one host plant showed phylogenetic clustering in host plant choice (Fig. 1) with the exception of Aricia artaxerxes (2 host plants; 
ses.mpd $=0.25 ;$ p-value $=0.43)$, Boloria napaea (2 host plants; ses.mpd $=0.26 ;$ p-value $=0.38)$, Callophrys rubi ( 8 host plants; ses.mpd $=-1.77$; p-value $=0.06$ ), Erebia pluto ( 2 host plants; ses.mpd $=-2.08$; $\mathrm{p}$-value $=0.08$ ) and Plebejus argus ( 6 host plants; ses.mpd $=-1.04 ; \mathrm{p}$-value $=0.12$ ). In addition, the interaction matrix structure was significantly correlated with the plant and butterfly phylogenies in the trophic interaction model (phylogenetic regressions: plants latent traits 1 and 2: Pagel's- $\lambda=0.8217$, p-value $<0.001$; Pagel's- $\lambda=0.6497, \quad$-value $<0.001$, and for butterfly latent traits 1 and 2: Pagel's- $\lambda=0.7257$, p-value $<0.001$; Pagel's- $\lambda=0.9230$, p-value $<0.001$, Pellissier et al. 2013a, b). This supports the view that herbivore diet show phylogenetic constraints, which is used to simulate diet expansion.

\section{Projections of abiotic and biotic models}

When considering the butterflies' abiotic projections, the median loss in area between 2010 and 2085 was $-21.5 \%$ (quantile 5\% $=-79.8 \%$, quantile $95 \%=185.1 \%$ ), $-64.8 \%$ $(-98.9 \%, 163.1 \%)$ and $-72.7 \%(-99.9 \%, 170.5 \%)$ under the RCP3PD, A1B and A2 scenarios, respectively. The percentage of species at risk of extinction with a predicted distribution area $<5 \%$ reached 10, 32 and 38\% (Supplementary material Appendix 1, Table A2).

Constraining the butterfly abiotic distribution with the distribution of their known host plants, lead to more restricted butterflies current (median $=-19 \%$, quantile $5 \%=-0.2 \%$, quantile $95 \%=-92.8 \%)$ and future projections (RCP3PD: median $=-15.3 \%$, quantile $5 \%=-92.3 \%$, quantile $95 \%=-0.9 \%$; A1B: $-17.3 \%,-98.8 \%,-0.3 \%$; A2: $-22.6 \%,-99.7 \%,-0.3 \%)$ compared to abiotic models. The median surface loss between current and 2085 biotic

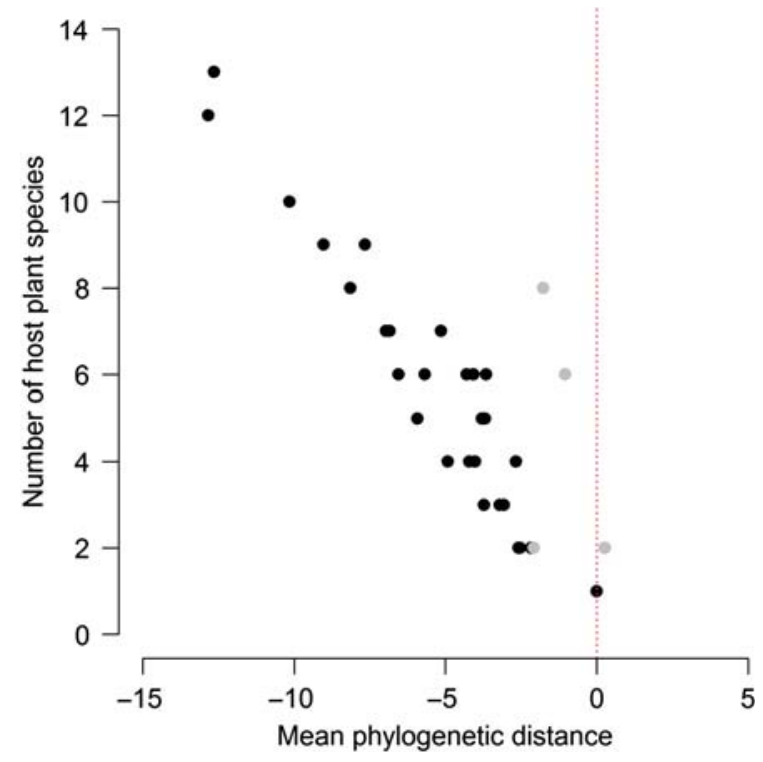

Figure 1. Biplot of the number of host plants in the diet of each butterfly species together with the standardized effect size of the mean phylogenetic distance (ses.mpd) between the host plants for each butterfly species. Negative values of ses.mpd indicate a phylogenetic clustering in host plant preference. Non-significant standardized effect sizes are represented by grey points. projections reached $-25.2 \%$ (quantile $5 \%=-84.6 \%$, quantile $95 \%=229.1 \%),-76.8 \%(-99.4 \%, 194.6 \%)$ and $-87 \%$ (-99.9\%, 193\%) under the RCP3PD, A1B and A2 scenarios, respectively. Constraining the butterfly abiotic distribution by known current trophic interaction increased the percentage of species at risk of extinction in 2085 from 10 to $20 \%$ for RCP3PD, from 32 to $52 \%$ for A1B and from 38 to $60 \%$ for A2 (Supplementary material Appendix 1, Table A2) and modified future butterfly species richness in the area (Supplementary material Appendix 1, Fig. A6).

\section{Response to diet expansion}

Simulations of butterfly host plant diet expansion under climate change showed a concave upward decreasing function in the relationship between degree of expansion and percent of species with risk of local extinction (extinction threshold 5\%: Fig. 2). Changing the selected extinction threshold to 1 or $10 \%$ of the total available open habitats did not change the shape of the curve (Supplementary material Appendix 1, Fig. A7, A8, Table A2). In contrast to a linear relationship or concave downward relationship, the decreasing concave upward shape indicates that diet expansion to even a few closely phylogenetically related host plants can significantly decrease the extinction rates. A conservative diet expansion to plants with interaction probabilities higher than 0.9 (number of species in the diet: mean $=14.9, \mathrm{SD}=16.5$ ) reduced the percent of butterfly species with extinction risk under climate change from 20 to $19 \%$ for RCP3PD, from 52 to $46 \%$ for $\mathrm{A} 1 \mathrm{~B}$ and from 60 to $51 \%$ for A2 (Fig. 2a, c, e, and Supplementary material Appendix 1, Table A2), decreased the number of species presenting range loss from 66 to $58 \%$ for RCP3PD, from 69 to $66 \%$ for A1B and from 71 to $67 \%$ for A2 (Supplementary material Appendix 1, Fig. A9a), and increased the forecasted butterfly species richness in cells (Fig. 3). Similarly, enlarging the diet of the butterfly to plants with a phylogenetic distance lower than 20 myr (number of species in the diet: mean $=8.9, S D=8.9$ ) reduced the percent of butterfly species with high extinction risk under climate change from 20 to $18 \%$ for RCP3PD, from 52 to $43 \%$ for $\mathrm{A} 1 \mathrm{~B}$ and from 60 to $48 \%$ for $\mathrm{A} 2$ (Fig. 2b, d, f) and decreased the number of species presenting range loss from 66 to $60 \%$ for RCP3PD, from 69 to $65 \%$ for A1B and from 71 to $67 \%$ for A2 (Supplementary material Appendix 1, Fig. A9b). Considering an unlikely diet expansion to all plants (interaction probability thresholds $=0$ and phylogenetic distance $=270$ ) corresponds to the abiotic projections presented above.

The scenario of diet expansion based on the interaction probability matrix was more restrictive than the one based purely on phylogenetic distance as shown by the intermediate values of the axis (Fig. 2). Enlarging the diet of the butterfly to plants with an interaction probability higher than 0.5 (number of species in the diet: mean $=21.5, S D=20.5$ ) reduced the percent of butterfly species with high extinction risk under climate change from 20 to $15 \%$ for RCP3PD, from 52 to $41 \%$ for A1B and from 60 to $49 \%$ for A2 (Fig. 2a, c, e, Fig. 3 and Supplementary material Appendix 1, Table A2) and decreased the number of species presenting range loss 
(a)

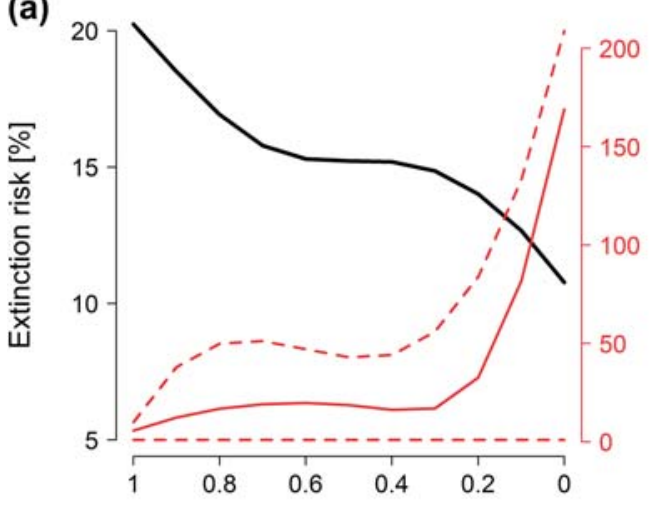

(c)

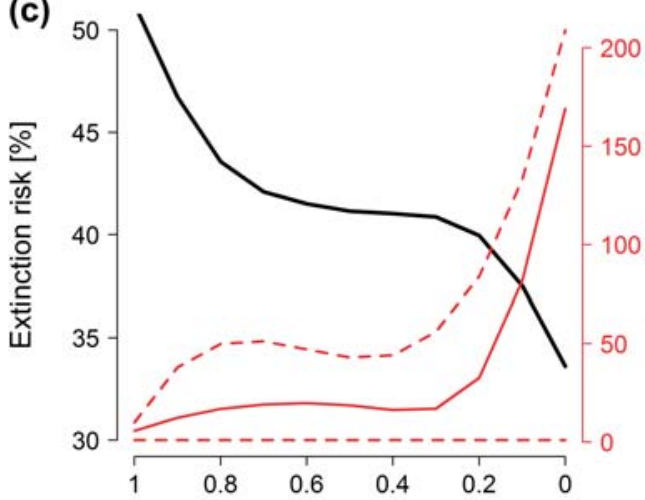

(e)

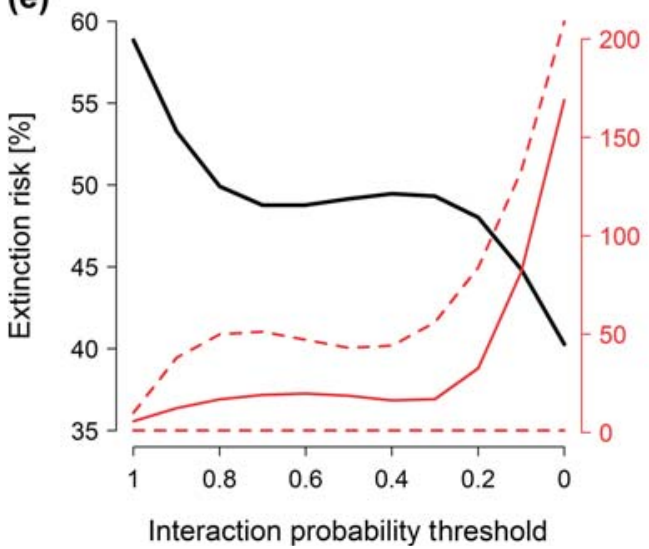

(b)

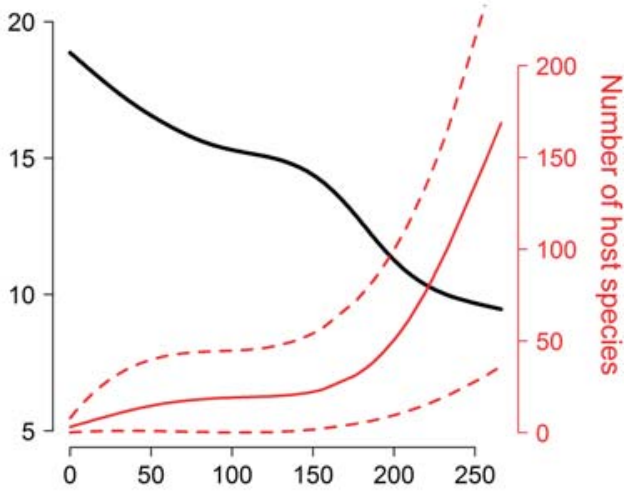

(d)

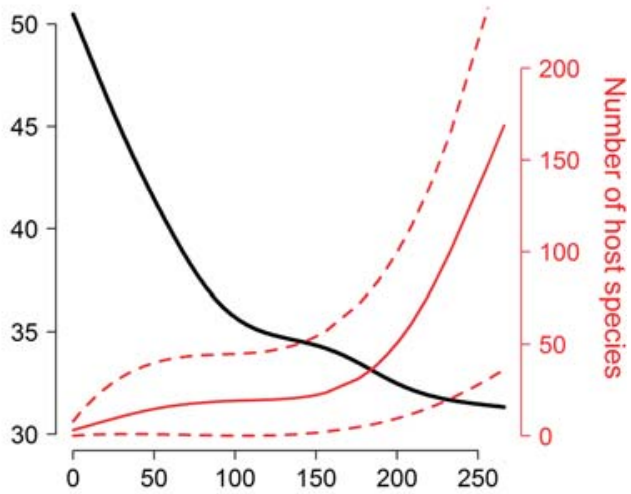

(f)

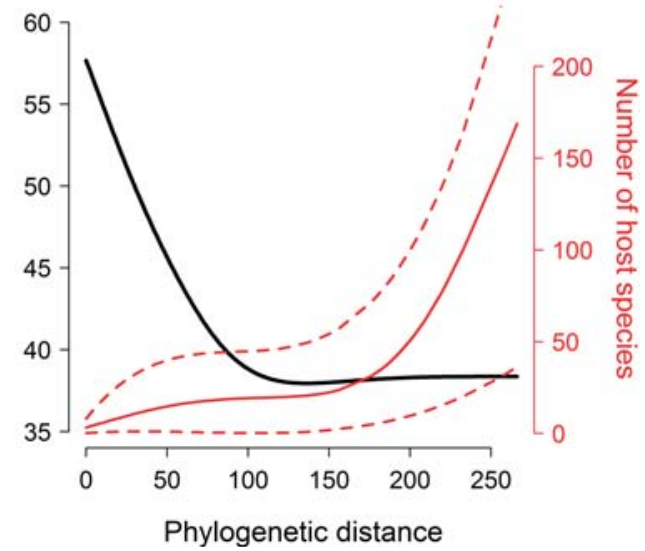

Figure 2. Percent of butterfly species with high extinction risk (black line) for the 2085 RCP3PD (a and b), A1B (c and d), A2 (e and f) climate change scenarios when considering host plants diet expansion based on ( $\mathrm{a}, \mathrm{c}$ and $\mathrm{e}$ ) modelled interaction probabilities between the butterfly and the plant and (b, $\mathrm{d}$ and $\mathrm{f}$ ) phylogenetic distances between plants. The mean accumulated number of host plants (red line) included in the diet is shown on the right axis (number of host species) where the dashed lines represent the 5 and 95 percentiles. An interaction probability threshold of 1 and a phylogenetic distance of 0 means that only the known host plants from the literature are considered in the diet. Curves were fitted with a GAM function.

from 66 to $56 \%$ for RCP3PD, from 69 to $66 \%$ for A1B and from 71 to $66 \%$ for A2 (Supplementary material Appendix 1, Fig. A9a). In contrast, enlarging the diet of the butterfly species to plants with a phylogenetic distance lower than 135 myr (number of species in the diet: mean $=22.5$, $\mathrm{SD}=15$ ) reduced the percent of butterfly species with high extinction risk under climate change for 2085 from 20 to $15 \%$ for RCP3PD, from 52 to $35 \%$ for A1B and from 60 to $38 \%$ for A2 (Fig. 2b, d, f) and decreased the number of species presenting range loss from 66 to $42 \%$ for RCP3PD, from 69 to $58 \%$ for $\mathrm{A} 1 \mathrm{~B}$ and from 71 to $63 \%$ for $\mathrm{A} 2$ (Supplementary material Appendix 1, Fig. A9b).

\section{Discussion}

Currently, species are tracking suitable abiotic conditions under climate change (Parmesan et al. 1999, Hickling et al. 2006), but future persistence will also depend on the availability of trophic resources (Araújo and Luoto 2007, Van der 
(a)

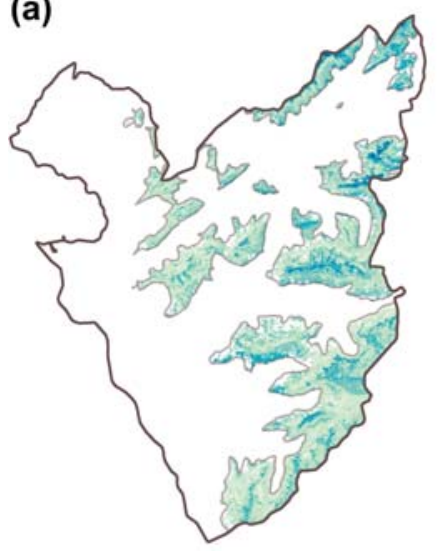

(b)

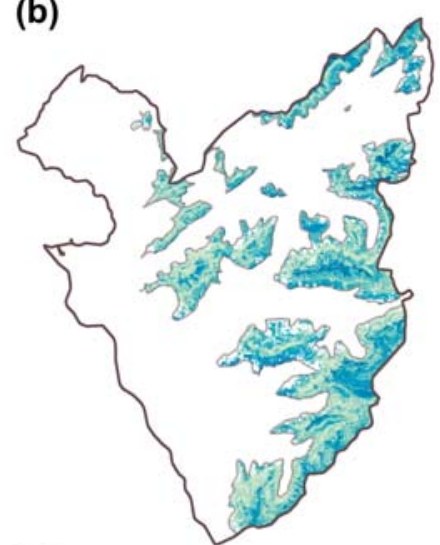

(d)

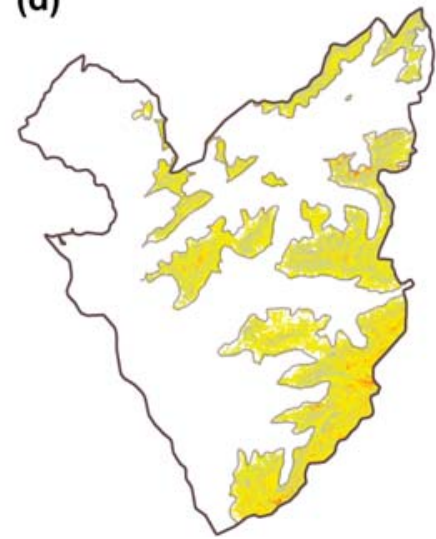

(c)

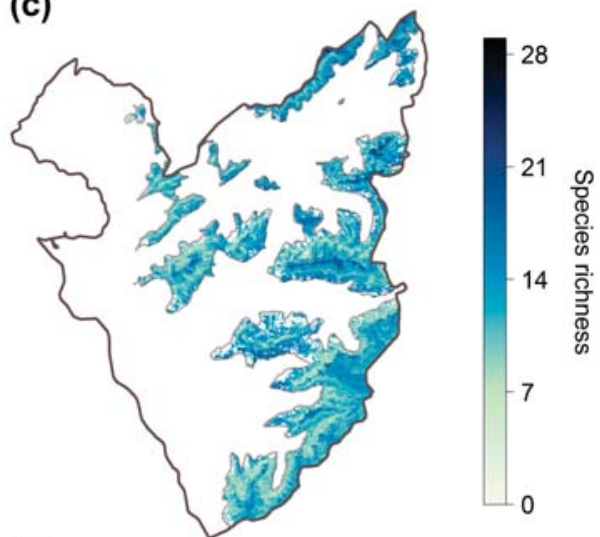

(e)

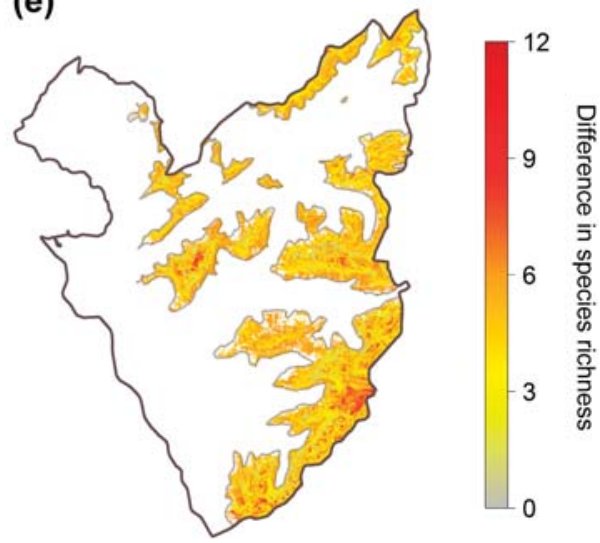

Figure 3. Predicted future (i.e. 2085) butterfly species richness in the study area in response to different diet expansion scenarios. The butterfly abiotic distribution was constrained with the abiotic distribution of host plants under the A2 climate change scenario when considering (a) only their known host plants, (b) a diet expansion scenario based on butterfly-plant interaction probabilities $>0.9$ and (c) a diet expansion scenario based on butterfly-plant interaction probabilities $>0.5$. (d) and (e) represent the changes in species richness induced by (d) a diet expansion scenario based on butterfly-plant interaction probabilities $>0.9$ (i.e. b minus a) and (e) a diet expansion scenario based on butterfly-plant interaction probabilities $>0.5$ (i.e. c minus a). To avoid problems of truncated response curves, projections are shown only over an elevation of $1600 \mathrm{~m}$, as a conservative estimate of the thermal elevational shifts under the most extreme climate change scenario.

Putten et al. 2010, Schweiger et al. 2012). The emergence of new trophic interactions may influence species response to climate change (Pateman et al. 2012, Blois et al. 2013). We show that the response of the number of species at risk of local extinction to simulated diet expansion follow a concave upward decreasing function (Fig. 2). The shape of the response curve suggests that the possibility to feed on even a few new host plants has a rapid attenuation effect - hereby the 'rescue effect' - on the percentage of species with high extinction risk (Fig. 3). Novel interaction may reduce species extinction risk, potentially supporting a rapid effect of adaptive responses on species persistence under climate change (Thuiller et al. 2013a).

While diet expansion in herbivores is possible within a short time period ( $<75$ yr; Strauss et al. 2006, Pateman et al. 2012), novel trophic interactions expected under climate change or invasions are difficult to predict (Pearse and Altermatt 2013). Statistical models of trophic interactions likelihood (Cattin et al. 2004, Pellissier et al. 2013a, b, Albouy et al. 2014, Rohr and Bascompte 2014) may be used to model the effects of likely novel interactions under climate change. Using the whole range of diet expansion possibilities, we studied the shape of the response between species at risk of extinction and diet expansion. The concave upward decreasing curve found using both approaches (i.e. interactions matrix and direct phylogenetic distance) suggests that even a low degree of diet expansion may reduce the number of species at risk of extirpation from the regional fauna. Our results in an alpine region contrast with an assessment at European scale, where only few species where limited by host plant distribution under climate change (Schweiger et al. 2012). This argues for considering species adaptive potential related to biotic interactions when forecasting species responses to climate change at least at smaller scale (Van der Putten et al. 2010, Thuiller et al. 2013a).

We found differences in the two diet expansion approaches considered. While the phylogenetic distance approach was solely based on the host plant phylogeny, the modelled interaction probability matrix was based on information provided by host plant and butterfly phylogenies together with plant species traits (i.e. leaf nitrogen; Pellissier et al. 2013a, b). This more ecologically constrained model showed a lower mitigating effect compared to the simulation based directly on phylogenetic distance (Fig. 2). While many secondary metabolites are phylogenetically conserved on plant clades (Wink 2003), other plant traits such as physical defense or leaf resource content are less conserved (Ndiribe et al. 2013) and may also modulate plant-herbivore interactions 
(Pellissier et al. 2012a, b, Ibanez et al. 2013). Integration of trophic interactions within future projection should provide more realistic models of species interactions including the relevant ecological traits modulating species interactions (Pellissier et al. 2013a, b, Ibanez et al. 2013, Albouy et al. 2014, Morales-Castilla et al. 2015).

Even when considering the potential adaptation of butterflies to new host plants, the percent of species with high extinction risk remained high. Thus, diet expansion to new host plants will not be able to completely mitigate the loss of suitable habitats due to climate change. In mountain areas, tracking suitable climatic conditions is not a long lasting solution, since suitable surfaces decrease upward the elevation gradient (Theurillat and Guisan 2001). The most coldadapted species currently restricted to alpine habitats, such as Plebejus glandon, are forecasted to become extinct regionally irrespective of host plant diet expansion due to the lack of suitable cold habitats in a warmer future. Even taking into account an unlikely diet expansion toward all available plant species in the study area, the number of species with high extinction risk remained elevated, at an estimated proportion of $10 \%$ for RCP3PD, 32\% for A1B and 38\% for A2 (Fig. 2). Adaptive responses to new host plants may thus attenuate the risk of extinction of butterfly species, but only to a limited degree while climate change may still largely drive species range shifts and range contraction (Schweiger et al. 2012).

The current study could be improved in several aspects. For simplifications, we considered unlimited dispersal for both host plants and butterfly species. While unlimited dispersal for butterflies might be realistic given their current observed range shift (Eskildsen et al. 2013, 2015), plants dispersal is slower than that of herbivore species (Rasmann et al. 2014). Adding a dispersal kernel for plants when tracking climate change in the landscape might provide more realistic assessment of range mismatches. Furthermore, studies have shown that soil variables (Dubuis et al. 2013) or land-use information (Randin et al. 2009, Schweiger et al. 2012) might also drive host plant distribution, so that future projections should consider refined environmental variables to provide realistic assessments. Finally, in the current study, we assumed that the host plant species list obtained from the literature is exhaustive (Pellissier et al. 2013a, b), but the literature may be incomplete. In such case, the 'no expansion' scenario might be too restrictive especially for more generalist species were omissions are more likely (Pellissier et al. 2013a, b).

In this study, we forecasted butterfly species distributions under climate change considering possible adaptation and demonstrated that changes in biotic interactions may modulate species responses. Mitigation effects through the evolutionary and/or ecological capacity of butterflies to enlarge their diet to new host plants might be a possible mechanism to prevent some species from local extinction. While new interactions under climate change have been documented, it remains to be seen whether trophic changes are common enough to make a difference. The understanding and prediction of interactions between herbivores and host plants using functional traits remain at an early stage (Ibanez et al. 2013, Pellissier et al. 2013a, b). Further studies of the mechanistic and chemical coupling between plant and herbivores are required to forecast future species interactions under climate change and refine the host plant diet expansion scenario developed in this study.

Acknowledgements - We thank the reviewers, especially Oliver Schweiger for highly valuable comments. We would like to thank Alexander Von Ungern, Anne Dubuis, Pascal Vittoz, Virginie Favre, Valéry Udry, Sarah Giovanettina, Vanessa Rion, Christian Purro, Saskia Godat and Aurore Gelin for field assistance. We thank Glenn Litsios for his help with the phylogenetic analysis. This study was supported by the European Commission (ECOCHANGE project, contract no. FP6 2006 GOCE 036866) and by the Swiss National Science Foundation (SNSF) grants no. 31003A-125145 (BIOASSEMBLE project) and no. 31003A-152866 (SESAM'ALP project) accorded to AG.

\section{References}

Albouy, C. et al. 2014. From projected species distribution to food-web structure under climate change. - Global Change Biol. 20: 730-741.

Allouche, O. et al. 2006. Assessing the accuracy of species distribution models: prevalence, kappa and the true skill statistic (TSS). - J. Appl. Ecol. 43: 1223-1232.

Araújo, M. B. and Luoto, M. 2007. The importance of biotic interactions for modelling species distributions under climate change. - Global Ecol. Biogeogr. 16: 743-753.

Becerra, J. X. 1997. Insects on plants: macroevolutionary chemical trends in host use. - Science 276: 253-256.

Blois, J. L. et al. 2013. Climate change and the past, present, and future of biotic interactions. - Science 341: 499-504.

Boggs, C. L. and Inouye, D. W. 2012. A single climate driver has direct and indirect effects on insect population dynamics. Ecol. Lett. 15: 502-508.

Bosshard, T. et al. 2011. Spectral representation of the annual cycle in the climate change signal. - Hydrol. Earth Syst. Sci. 15: 2777-2788.

Brooker, R. W. et al. 2007. Modelling species' range shifts in a changing climate: the impacts of biotic interactions, dispersal distance and the rate of climate change. - J. Theor. Biol. 245: 59-65.

Cattin, M. F. et al. 2004. Phylogenetic constraints and adaptation explain food-web structure. - Nature 427: 835-839.

Devictor, V. et al. 2012. Differences in the climatic debts of birds and butterflies at a continental scale. - Nat. Clim. Change 2: $121-124$

Dubuis, A. et al. 2011. Predicting spatial patterns of plant species richness: a comparison of direct macroecological and species stacking modelling approaches. - Divers. Distrib. 17: $1122-1131$

Dubuis, A. et al. 2013. Improving the prediction of plant species distribution and community composition by adding edaphic to topo-climatic variables. - J. Veg. Sci. 24: 593-606.

Elith, J. et al. 2006. Novel methods improve prediction of species' distributions from occurrence data. - Ecography 29: 129-151.

Engler, R. and Guisan, A. 2009. MigClim: predicting plant distribution and dispersal in a changing climate. - Divers. Distrib. 15: 590-601.

Engler, R. et al. 2011. 21st century climate change threatens mountain flora unequally across Europe. - Global Change Biol. 17: $2330-2341$.

Eskildsen, A. et al. 2013. Testing species distribution models across space and time: high latitude butterflies and recent warming. - Global Ecol. Biogeogr. 22: 1293-1303.

Eskildsen, A. et al. 2015. Ecological specialization matters: long-term trends in butterfly species richness and assemblage composition depend on multiple functional traits. - Divers. Distrib. 21: 792-802. 
Fielding, A. H. and Bell, J. F. 1997. A review of methods for the assessment of prediction errors in conservation presence/ absence models. - Environ. Conserv. 24: 38-49.

Friedman, J. H. 2001. Greedy function approximation: a gradient boosting machine. - Ann. Stat. 29: 1189-1232.

Geiger, W. 1987. Les papillons de jour et leurs biotopes. - Ligue Suisse pour la protection de la Nature, Bâle, Switzerland.

Gilman, S. E. et al. 2010. A framework for community interactions under climate change. - Trends Ecol. Evol. 25: 325-331.

Guisan, A. and Thuiller, W. 2005. Predicting species distribution: offering more than simple habitat models. - Ecol. Lett. 8: 993-1009.

Hanley, J. A. and Mcneil, B. J. 1982. The meaning and use of the area under a receiver operating characteristic (ROC) curve. - Radiology 143: 29-36.

Hastie, T. and Tibshirani, R. 1990. Generalized additive models. - Stat. Sci. 1: 297-318.

Hickling, R. et al. 2006. The distributions of a wide range of taxonomic groups are expanding polewards. - Global Change Biol. 12: 450-455.

Hirzel, A. and Guisan, A. 2002. Which is the optimal sampling strategy for habitat suitability modelling. - Ecol. Model. 157: 331-341.

Ibanez, S. et al. 2013. Herbivory mediated by coupling between biomechanical traits of plants and grasshoppers. - Funct. Ecol. 27: 479-489.

Kearney, M. et al. 2009. Integrating biophysical models and evolutionary theory to predict climatic impacts on species' ranges: the dengue mosquito Aedes aegypti in Australia. - Funct. Ecol. 23: 528-538.

Kembel, S. W. et al. 2010. Picante: R tools for integrating phylogenies and ecology. - Bioinformatics 26: 1463-1464.

Kissling, W. D. et al. 2012. Towards novel approaches to modelling biotic interactions in multispecies assemblages at large spatial extents. - J. Biogeogr. 39: 2163-2178.

Lavergne, S. et al. 2010. Biodiversity and climate change: integrating evolutionary and ecological responses of species and communities. - Annu. Rev. Ecol. Evol. Syst. 41: 321-350.

Liu, C. et al. 2005. Selecting thresholds of occurrence in the prediction of species distributions. - Ecography 28: 385-393.

McCullagh, P. and Nelder, J. A. 1989. Generalized linear models. - Chapman and Hall.

Merrill, R. M. et al. 2008. Combined effects of climate and biotic interactions on the elevational range of a phytophagous insect. - J. Anim. Ecol. 77: 145-155.

Morales-Castilla, I. et al. 2015. Inferring biotic interactions from proxies. - Trends Ecol. Evol. 30: 347-356.

Morin, X. and Chuine, I. 2006. Niche breadth, competitive strength and range size of tree species: a trade-off based framework to understand species distribution. - Ecol. Lett. 9: 185-195.

Morin, X. and Thuiller, W. 2009. Comparing niche- and process-based models to reduce prediction uncertainty in species range shifts under climate change. - Ecology 90: 1301-1313.

Ndiribe, C. et al. 2013. Phylogenetic plant community structure along elevation is lineage specific. - Ecol. Evol. 3: 4925-4939.

Nogués-Bravo, D. and Rahbek, C. 2011. Communities under climate change. - Science 334: 1070-1071.

Parmesan, C. 2006. Ecological and evolutionary responses to recent climate change. - Annu. Rev. Ecol. Evol. Syst. 37: 637-669.

Parmesan, C. et al. 1999. Poleward shifts in geographical ranges of butterfly species associated with regional warming. - Nature 399: 579-583.

Pateman, R. M. et al. 2012. Temperature-dependent alterations in host use drive rapid range expansion in a butterfly. - Science 336: 1028-1030.

Pearse, I. S. and Altermatt, F. 2013. Predicting novel trophic interactions in a non-native world. - Ecol. Lett. 16: $1088-1094$.
Pellissier, L. et al. 2012a. Shifts in species richness, herbivore specialization, and plant resistance along elevation gradients. - Ecol. Evol. 2: 1818-1825.

Pellissier, L. et al. 2012b. Climate-based empirical models show biased predictions of butterfly communities along environmental gradients. - Ecography 35: 684-692.

Pellissier, L. et al. 2013a. Turnover of plant lineages shapes herbivore phylogenetic beta diversity along ecological gradients. - Ecol. Lett. 16: 600-608.

Pellissier, L. et al. 2013b. Combining food web and species distribution models for improved community projections. - Ecol. Evol. 3: 4572-4583.

Pollard, E. and Yates, T. J. 1993. Monitoring butterflies for ecology and conservation. - Chapman and Hall.

Pöyry, J. et al. 2009. Species traits explain recent range shifts of Finnish butterflies. - Global Change Biol. 15: 732-743.

Pradervand, J.-N. et al. 2014. Functional homogenization of bumblebee communities in alpine landscapes under projected climate change. - Clim. Change Responses 1: 1-10.

Randin, C. F. et al. 2009. Introduction of snow and geomorphic disturbance variables into predictive models of alpine plant distribution in the western Swiss Alps. - Arct. Antarct. Alp. Res. 41: 347-361.

Rasmann, S. et al. 2014. Climate-driven change in plant-insect interactions along elevation gradients. - Funct. Ecol. 28: 46-54.

Reznick, D. N. and Ghalambor, C. K. 2001. The population ecology of contemporary adaptations: what empirical studies reveal about the conditions that promote adaptive evolution. - Genetica 112-113: 183-198.

Ridgeway, G. 1999. The state of boosting. - Comput. Sci. Stat. 31: 172-181.

Rohr, R. P. and Bascompte, J. 2014. Components of phylogenetic signal in antagonistic and mutualistic networks. - Am. Nat. 184: 556-564.

Roland, J. and Matter, S. F. 2013. Variability in winter climate and winter extremes reduces population growth of an alpine butterfly. - Ecology 94: 190-199.

Romo, H. et al. 2014. Effects of climate change on the distribution of ecologically interacting species: butterflies and their main food plants in Spain. - Ecography 37: 1063-1072.

Schweiger, O. et al. 2008. Climate change can cause spatial mismatch of trophically interracting species. - Ecology 89: 3472-3479.

Schweiger, O. et al. 2010. Multiple stressors on biotic interactions: how climate change and alien species interact to affect pollination. - Biol. Rev. Camb. Phil. Soc. 85: 777-795.

Schweiger, O. et al. 2012. Increasing range mismatching of interacting species under global change is related to their ecological characteristics. - Global Ecol. Biogeogr. 21: 88-99.

Strauss, S. Y. et al. 2006. Evolutionary responses of natives to introduced species: what do introductions tell us about natural communities? - Ecol. Lett. 9: 357-374.

Theurillat, J. P. and Guisan, A. 2001. Potential impact of climate change on vegetation in the European Alps: a review. - Clim. Change 50: 77-109.

Thuiller, W. et al. 2005. Climate change threats to plant diversity in Europe. - Proc. Natl Acad. Sci. USA 102: $8245-8250$.

Thuiller, W. et al. 2009. BIOMOD - a platform for ensemble forecasting of species distributions. - Ecography 32: 369-373.

Thuiller, W. et al. 2013a. A road map for integrating eco-evolutionary processes into biodiversity models. - Ecol. Lett. 16 (Suppl. 1): 94-105.

Thuiller, W. et al. 2013b. biomod2: ensemble platform for species distribution modeling. - $\mathrm{R}$ package ver. 3.1-18, $<$ http://CRAN.R-project.org $>$. 
Van der Putten, W. H. et al. 2010. Predicting species distribution and abundance responses to climate change: why it is essential to include biotic interactions across trophic levels. - Phil. Trans. R. Soc. B 365: 2025-2034.

Walther, G.-R. et al. 2010. Ecological repsonses to recent climate change. - Nature 416: 389-395.

Wilson, R. J. et al. 2007. An elevational shift in butterfly species richness and composition accompanying recent climate change. - Global Change Biol. 13: 1873-1887.

Supplementary material (Appendix ECOG-01557 at <www.ecography.org/appendix/ecog-01557>). Appendix 1.
Wink, M. 2003. Evolution of secondary metabolites from an ecological and molecular phylogenetic perspective. - Phytochemistry 64: 3-19.

Wisz, M. S. et al. 2013. The role of biotic interactions in shaping distributions and realised assemblages of species: implications for species distribution modelling. - Biol. Rev. Camb. Phil. Soc. 88: 15-30.

Zimmermann, N. E. and Kienast, F. 1999. Predictive mapping of alpine grasslands in Switzerland: species versus community approach. - J. Veg. Sci. 10: 469-482. 